\title{
A CHARACTERIZATION OF THE SUBGROUPS OF FINITELY PRESENTED GROUPS ${ }^{1}$
}

\author{
BY GEORGE S. SACERDOTE
}

Communicated by R. M. Fossum, February 16, 1976

\begin{abstract}
We characterize the subgroups of finitely presented groups as those countable groups having consistently recursively enumerable word problem. We also characterize the subgroups of simple subgroups of finitely presented groups as those countable groups having consistently recursive word problem.
\end{abstract}

Graham Higman has characterized the finitely generated subgroups of finitely presented groups as those finitely generated groups with recursively enumerable (r.e.) word problem (see $[\mathbf{H}]$ ). In the same paper he asked for a characterization of all countable groups which are subgroups of finitely presented groups. Theorem I below gives such a characterization.

Definition 1. A countable group $G$ has r.e. word problem relative to a set $S$ of generators if and only if there is a partial algorithm which converges on all words on $S$ which equal 1 in $G$ and gives answer "yes", and does not give answer "yes" on any word $\neq 1$ in $G$. (It could not converge, or it could converge and answer "no".) If the word problem for $G$ is r.e. relative to some generating set $S$, we will say that $G$ has r.e. word problem.

DEFINITION 2. $G$ has recursive word problem relative to $S$ if there is a partial algorithm which converges for all words $w$ on $S$, and answers correctly whether or not $w=1$ in $G$. If the word problem for $G$ is recursive relative to some generating set, we will say $G$ has solvable word problem.

Definition 3. If $A$ is a recursive alphabet and if $f$ is a partial algorithm from the set of words on $A$ to the set "yes", "no" we call $f$ a consistent algorithm if the set of conditions $\{W=1 \mid f(W)=$ "yes" $\} \cup\{W \neq 1 \mid f(W) \neq$ "yes" $\}$ is consistent with the axioms of group theory.

If $f$ is a consistent algorithm, then the set of words $W$ such that $f(W)=$ "yes" is closed under concatenation, and conjugation by arbitrary words $U$ on $A$. In group theoretic terms this means that $f(U)=$ "yes" if and only if $f\left(U^{\prime}\right)=$ "yes" where $U^{\prime}$ is the freely reduced word equivalent to $U$, and if $F$ is the free group on $A$, the set of equivalence classes of words $W$ such that $f(W)=$ "yes" is a normal subgroup of $F$.

AMS( MOS) subject classifications (1970). Primary 20F15, 20F10, $02 \mathrm{~F} 47$.

${ }_{1}^{1}$ The research for this paper was supported by NSF Grant MPS 75-07099. The author wishes to thank the Mathematics Department of the University of Illinois for its generous hospitality during part of the time in which the results reported were obtained. 
Definition 4. A group $G$ has consistently r.e. (or recursive) word problem if and only if its word problem is r.e. (or recursive) via a consistent algorithm.

It should be noted that a finitely generated group has consistently r.e. word problem (relative to any finite set of generators) if and only if it has r.e. word problem.

Henceforth, if we have a group $G$ with a set $S$ of generators, we will assume (without loss of generality) that

$$
S \subset A=\left\{a_{1}, a_{2}, \ldots\right\}
$$

THEOREM I. A countable group is embeddable into a finitely presented group if and only if the word problem for $G$ is consistently recursively enumerable.

Boone and Higman have characterized the finitely generated groups with solvable word problem as those finitely generated groups embeddable into simple subgroups of finitely presented groups (see [B4]). Theorem II below is a natural generalization of their result:

THEOREM II. A countable group is a subgroup of a simple subgroup of a finitely presented group if and only if it has consistently solvable word problem.

The author has given theorems (see $[\mathbf{S}]$ ) which characterize algebraically the finitely generated groups with solvable order, power, conjugacy or Magnus problems. All of these theorems have natural extensions to the cases of arbitrary countable groups in which these problems are consistently solvable.

Murskii (see [M]) has proved the analog of Higman's theorem, and Boone and Higman (see $[\mathbf{B H}]$ ) have proved the analog of their theorem, for finitely generated semigroups. Both of these theorems generalize in a natural way to the exact analogs of Theorems I and II above for semigroups.

Several further theorems can also be proved.

Definition 5. A countable group has locally r.e. (or recursive) word problem if every finitely generated subgroup has r.e. (or recursive) word problem.

Theorem III below relates these two notions to the notions introduced earlier in this announcement.

THEOREM III. (i) Every group whose word problem is locally recursively enumerable has recursively enumerable word problem.

(ii) There exists a group whose word problem is recursively enumerable but not consistently recursively enumerable.

(iii) There exists a group whose word problem is locally recursive but not recursive.

(iv) There exists a group whose word problem is recursive, but not consistently recursive. 


\section{BIBLIOGRAPHY}

[BH] William W. Boone and G. Higman, An algebraic characterization of groups with soluble word problem, J. Austral. Math. Soc. 18 (1974), 41-53. MR 50 \#10093.

[H] G. Higman, Subgroups of finitely presented groups, Proc. Roy. Soc. Ser. A 262 (1961), 455-475. MR 24 \#A152.

[M] V. L. Murskir, Isomorphic embeddability of semigroups with countable sets of defining relations in finitely defined semigroups, Mat. Zametki 1 (1967), 217-224 = Math. Notes 1 (1967), 145-149. MR 35 \#1686.

[S] Georege S. Sacerdote, On the Boone-Higman theorem and the conjugacy problem (to appear).

DEPARTMENT OF MATHEMATICS, AMHERST COLLEGE, AMHERST, MASSACHUSETTS 01002 